\title{
Tolerance Modeling Using the Feature Based Product Model for Prediction of Used Parts Deterioration
}

\author{
M. MATSUDA ${ }^{1}$, H. SUZUKI ${ }^{2}$ and F. KIMURA ${ }^{3}$ \\ ${ }^{1}$ Department of Information and Computer Sciences, Kanagawa Institute of Technology, 1030 \\ Shimo-ogino, Atsugi-shi, Kanagawa 243-0292, Japan, \\ Tel:+81-46-291-3213, Fax: +81-46-242-8490, e-mail: matsuda@ic.kanagawa-it.ac.jp, \\ ${ }_{2}^{2}$ Arthur D. Little (Japan), Inc. \\ ${ }^{3}$ Department of Precision Machinery Engineering, The University of Tokyo
}

\begin{abstract}
The objective of this paper is the prediction for the deteriorated status of mechanical parts based on the feature-oriented product model for supporting the parts reuse phase. As the first step, the product model with "Geometrical Quality Feature" is proposed for representing the deteriorated status. The geometrical quality feature consists of "form tolerance" and "geometricalrelation constraint". Based on the TTRS (Technologically and Topologically Related Surface) method, the form tolerance is described for representing deteriorated deformation of each geometric element. A geometrical-relation constraint is defined corresponding to the type of surfaces and its geometrical relation. The relationship between the form tolerances and the geometricalrelation constraint is also described in each movable element for interpreting the change of form tolerance into that of the geometrical-relation constraint. In the prediction process of parts deterioration using the geometrical quality feature, the effect of the deteriorated deformation on the functional behaviour is calculated using the cause list and behaviour simulation. This calculated status is assigned to each geometric element through form tolerance and it refers to the prediction of the deteriorated status comparing to the usage history. Through this process, the reuse of mechanical parts as a material for the machining process can be supported.
\end{abstract}

Key words: Life Cycle Engineering, Product Model, Tolerance Modelling, Prediction of Deterioration, Reuse of Parts

The original version of this chapter was revised: The copyright line was incorrect. This has been corrected. The Erratum to this chapter is available at DOI: 10.1007/978-0-387-35637-2_11 


\section{INTRODUCTION}

In order to solve such problems as the shortage of energy and natural resources and environmental pollution, a paradigm change of the present manufacturing system is required. One of the most effective solutions for these problems is to take the whole product life cycle into consideration at the product design phase and realize a closed-loop product life cycle. The product life cycle considered here includes an inverse flow from products to materials or parts. Products are decomposed into several parts after the use phase. Some of these are re-used at the production stage and during the maintenance/up-grade process. In order to reuse parts, their quality has to be predicted geometrically. In order to assist in the quality evaluation of used parts before reuse, a prediction of the status of deterioration with respect to geometrical information like its dimension and tolerance should also be made. In order to support the product life cycle in the view of product information, various product models have been proposed. However, they are mainly focused on the product design and manufacturing phase and its linkage. If the same product model could be used for the other life cycle phases like the prediction of deterioration before reuse, it would lead to a more efficient information exchange throughout the whole product life cycle.

The final objective of this paper is to support the reuse phase by constructing the additional feature of representing the deteriorated status in the feature-oriented product model and by predicting the geometrical quality of each part using this model (Suzuki 2000). In the following sections, the relationship between product life cycle and product model is considered and a geometrical quality feature is newly defined in order to represent the deteriorated status with the product model. The geometrical quality feature consists of form tolerances and geometrical-relation constraints. Furthermore the prediction system for parts deterioration based on the product model with geometrical quality feature is proposed. Finally, an example of this method is introduced to verify the feasibility of this method.

\section{PRODUCT MODEL FOR PARTS REUSE}

\subsection{Product life cycle and product model}

Due to the rapid development of information technology, product models have been proposed to integrate the various aspects of information about mechanical products (Krause 1993). With the aid of CAD tools, a geometryoriented product model is created during the product design phase. From the 
view of each life cycle phase, each phase has a corresponding different viewpoint and acquires necessary information from the same geometryoriented model. On the other hand, the life cycle of a certain product includes various phases such as planning, manufacturing, use and so on. Figure 1 shows this relationship between the life cycle flow for each product and the life cycle process, especially focused on the manufacturing phase.

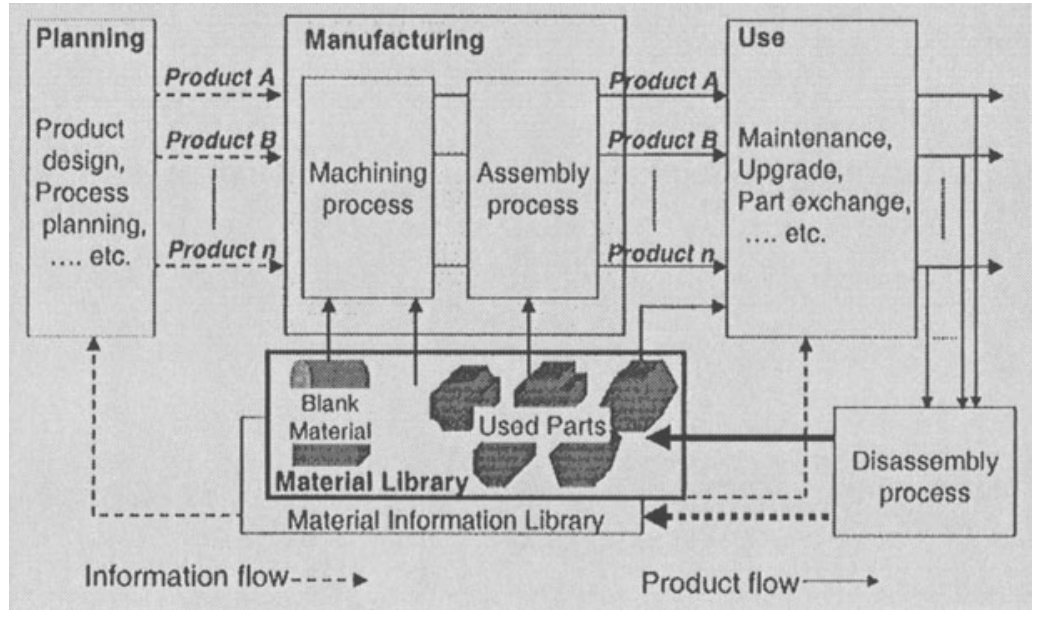

Figure 1. Product Life Cycle with part's reuse flow

In this paper, mechanical products are considered that are produced with large varieties of types and very small production volumes. In this type of production, it is important that used parts from a product can be accepted as materials for another product in the manufacturing phase. Therefore, the manufacturing phase should be focused as well as the reuse phase itself. In the manufacturing phase considering parts reuse, several types of parts from different products have to be processed in the same manufacturing process. The manufacturing process can be divided into machining process and assembly process. If only blank materials are used in the manufacturing process, only one machining process plan can be designed. However, if used parts from different products are also treated as candidates of machining in a material library, several processes should be considered. Because the deteriorated status of each used part can be different, one part may need to be refurbished while others may be able to be used as assembly parts without refurbishment. Also some parts may be used as repair parts in the maintenance process. Here, refurbishment means the additional machining required for remaking the parts into the parts for another product. Many of the mechanical products, which are considered in this paper, have already been intended to be used repeatedly by refurbishment if necessary. For this mixture of product life cycle lines, more complicated processes are required 
in the manufacturing phase (Matsuda 1999). From the viewpoint of energy consumption, reusing parts as materials for machining tends to consume less energy than in the case of producing from blank material. And during the machining phase, parts reuse makes it possible to decrease the amount of machining, which results in the saving of manufacturing resources. Therefore the parts reuse phase should be involved in the product life cycle of the mechanical products.

\subsection{Requirements of product model for parts reuse}

The product model provides information about the overall product shape, product size, finishing accuracy, relationship between parts and so on. In this paper, solid models are considered as the basis of the product model. During the product design phase, most of this information is generated. This information is described in the product model and used during other life cycle phases. However, the present product model is not necessarily amenable to support inverse life cycle phases such as parts reuse. In order to use the same product model in the reuse phase as well, the requirements for the product model should be re-considered. Figure 2 shows how the product model should be used for supporting the parts reuse phase.

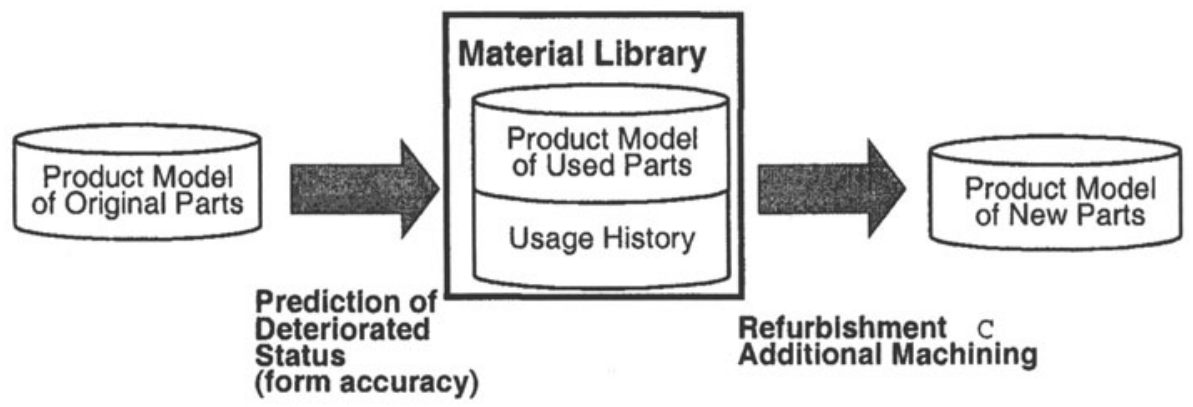

Figure 2. Parts reuse process and product model

The deteriorated condition of each used part is predicted as a change in accuracy of form based on the usage history. An altered product model based on the prediction of deterioration is added to a material library as a candidate for machining. If it matches requirements for another part, refurbishing process data for the selected candidate parts are generated based on the selected product model. Then this product model is again circulated as a new product model in the next life cycle. 
For supporting the parts reuse phase, the product model has to be able to be used in the quality management of used parts before its reuse. For this quality management, the product model has to fulfil the following three requirements. The first requirement is the ability to apply the prediction process of geometrical deteriorated status using the calculated method such as a behaviour simulation. As considered, a geometry-oriented product model is mainly applied to predict how a certain geometrical property, such as accuracy of form may have an effect on the product behaviour. In the product model, these geometrical accuracies are represented as the relationship between geometric elements such as surfaces. Therefore, if these properties are involved in the geometrical product model, they should be treated as parameters of the input model in the calculation process. The second requirement is the ability to add the result of the prediction into the original product model. In the present product model, it is hardly supposed that the information about geometrical quality should be added for the inverse process. In order to add the result of the prediction process, another attribute should be added to the normal product model. The third requirement is the ability to be used for production planning including refurbishment. Since such machining is done for each part, these geometrical accuracies have to be assigned to each geometric element from the refurbishment viewpoint.

\section{GEOMETRICAL QUALITY FEATURE}

From the discussion of the requirements of the product model for employment in the parts reuse phase, it was found that one of the most critical requirements is how to represent the geometrical accuracy in the product model. At present, tolerance concepts have important roles as an indicator of geometrical accuracy during the design, manufacturing and inspection phase of the new parts. Any dimension and shape of machined parts has some geometrical variations from nominal value and shape. While product functions have to be satisfied using parts with geometrical variations. Tolerance concepts have been introduced for showing the amount of tolerant variations from both the functional requirements and the machining cost. (Bjorke 1989) For this role, each tolerance is assigned to each independent part and the designer then sets a certain value and transmits the necessary machining accuracy from the design phase to the manufacturing phase. The present tolerance concept can be divided into dimensional tolerance and geometrical tolerance. The standard dimensional tolerance concept is widely used in the industry and plays an important role for the interchangeability of mechanical parts. However this concept leaves 
some ambiguities about the shape. On the other hand, geometrical tolerance concepts can give the strict definition of each geometrical form, position and orientation. The present tolerance definitions have not been shown to be general indicators of the geometrical quality at the inverse phase such as parts reuse. In this paper, this tolerance definition should be extended to include geometrical quality through the whole product life cycle. It means that a minute change of geometry due to such deterioration as wear and abrasion should be represented by this extended geometrical tolerance description. For these reasons, a geometrical quality feature is newly defined based on the extensive tolerance descriptions and is added to the product model. Figure 3 shows the structure of the product model with the geometrical quality feature.

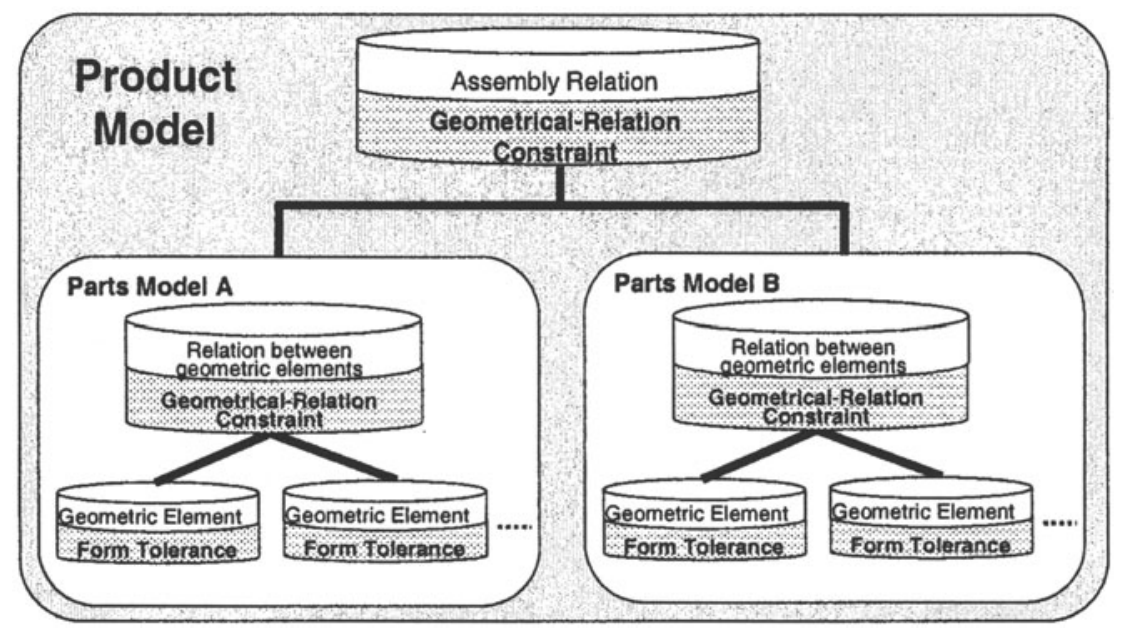

Figure 3. Feature based product model with geometrical quality feature

The geometrical quality feature consists of form tolerance descriptions and geometrical-relation constraint descriptions. Each form tolerance is described corresponding to a particular geometric element of a part's solid model. On the other hand, geometrical-relation constraints are described with positional and/or orientational relation that represents the connecting relationships between geometric elements. Geometrical-relation constraints are defined based on the present relative tolerance definition. The difference between the normal relative tolerance and this geometrical-relation constraint is that normal relative tolerance is defined between elements which are involved in one part, while geometrical-relation constraints can also be defined between different parts.

Figure 4 shows the role of geometrical quality feature from the product information process. In the mechanical product, many of product functions 
are realized through the mechanical behaviours. These behaviours are made up of the mechanical capability of each part as well as the part's role in the overall structure. Therefore the geometrical accuracy given by the geometrical variations of each part and its relation can have an effect on the behaviour. The main role of the geometrical quality feature is to relate the mechanical behaviours and the geometrical accuracies.



Figure 4. Relationship between product function and its geometry using geometrical quality feature

There are three advantages to be derived from the geometrical quality feature. The first advantage is that by changing the value of form tolerance and geometrical-relation constraint due to deterioration, the geometrical quality feature can be used as a parameter for a geometrical accuracy. The second advantage of this feature is to define the relationship between form tolerance and the geometrical-relation constraint. The third is that both form tolerance and geometrical-relation constraints are based on the definition of the present geometric tolerance, so that the exiting tolerance methods such as tolerance specification and tolerance analysis can be applied for specifying this feature and prediction process. 


\section{DESCRIPTION OF GEOMETRICAL QUALITY FEATURE}

\subsection{Description of form tolerance and geometrical- relation constraint}

In order to define form tolerance and the geometrical-relation constraint, TTRS (Technologically and Topologically Related Surfaces) (Salomons 1995) is introduced in this paper. In the TTRS method, any surface on the solid model can be divided into seven types based on the displacement that leaves a surface invariance; spherical, planar, cylindrical, helical, surfaces of revolution, prismatic and any surfaces. Table 3 shows these elementary surfaces of TTRS.

One of the biggest advantages of the TTRS method is that it is possible to define new TTRS repeatedly between two TTRS according to the invariance. So TTRS can be applied not only to the real surface involved in a solid model but also to the relative geometry, which is called Pseudo_TTRS (Clement 1995). This means a certain geometrical-relation constraint between different parts can be represented using the same definition of a standard relative tolerance such as parallelism, coaxiality and so on. Based on this repetitive TTRS definition, a proper type of geometrical-relation constraint can be inferred. Specific form tolerances can be defined for each elementary surface of TTRS in order to describe its complete shape. If the shape of parts has been changed by such deterioration as abrasion, this change is interpreted as the change of the value of the form tolerance. Each elementary surface of TTRS can be also applied to specific relative tolerance types that can be defined in the TTRS relation involved. In this paper, this is defined as a geometrical-relation constraint based on the relation between the involved TTRS. Table 1 also shows definable geometrical-relation constraints and form tolerances for each elementary surface.

\subsection{Description of movable element}

To represent the geometrical deteriorated status using the geometrical quality feature, the very part, which had caused the deformation by deterioration, should be paid attention to. As relative motions between contacting geometric elements cause many of the geometrical deteriorations such as wear in a mechanical product, in this paper it is assumed that the deformation caused by deterioration occurs only to movable parts. Therefore, it is important how movable elements are modelled using the geometrical quality feature. In the mechanical product, there are some 
typical elements for movement. Table 2 shows some examples of these typical movable elements (Suzuki 2000).

Table 1: Elementary surface of TTRS and its definable tolerance

\begin{tabular}{|c|c|c|}
\hline $\begin{array}{l}\text { Elementary } \\
\text { Surface of TTRS }\end{array}$ & Definable Form Tolerance & $\begin{array}{l}\text { Definable Relative Tolerance } \\
\text { (Geometrical-relation constraint) }\end{array}$ \\
\hline Sphere & Circularity & Coaxiality \\
\hline Plane & Flatness, Straightness & $\begin{array}{l}\text { Parallelism, Perpendicularity, } \\
\text { Position }\end{array}$ \\
\hline Cylindrical & $\begin{array}{l}\text { Cylindricity, Circularity, } \\
\text { Straightness (Axis) }\end{array}$ & $\begin{array}{l}\text { Coaxiality, Parallelism, } \\
\text { Perpendicularity, Position }\end{array}$ \\
\hline Helical & (None) & $\begin{array}{l}\text { Coaxiality, Parallelism, } \\
\text { Perpendicularity, Position }\end{array}$ \\
\hline Of revolution & $\begin{array}{l}\text { Profile of surface, Circularity, } \\
\text { Straightness (Axis), Profile of line }\end{array}$ & $\begin{array}{l}\text { Coaxiality, Parallelism, } \\
\text { Perpendicularity, Position }\end{array}$ \\
\hline Prismatic & $\begin{array}{l}\text { Profile of surface, Straightness } \\
\text { (Axis), Profile of line }\end{array}$ & $\begin{array}{l}\text { Coaxiality, Parallelism, } \\
\text { Perpendicularity, Position }\end{array}$ \\
\hline General & Profile of surface, Profile of line & (None) \\
\hline
\end{tabular}

Table 2: Example of common movable elements

\begin{tabular}{|c|c|c|c|c|}
\hline $\begin{array}{l}\text { Example of } \\
\text { Movable Elements }\end{array}$ & $\begin{array}{l}\text { Combination } \\
\text { of Surface } \\
\text { Type }\end{array}$ & $\begin{array}{l}\text { Type of } \\
\text { Geometrical- } \\
\text { relation Constraint }\end{array}$ & $\begin{array}{l}\text { Type of } \\
\text { Movement }\end{array}$ & $\begin{array}{l}\text { Applied } \\
\text { Form } \\
\text { Tolerance }\end{array}$ \\
\hline Feed & $\begin{array}{l}\text { Cylinder - } \\
\text { Plane }\end{array}$ & Parallelism & $\begin{array}{l}\text { Translation } \\
\text { [Plane] + Rotation } \\
\text { [Cylinder] }\end{array}$ & $\begin{array}{l}\text { Flatness, } \\
\text { Cylindricity }\end{array}$ \\
\hline Crank & $\begin{array}{l}\text { Cylinder - } \\
\text { Cylinder }\end{array}$ & Coaxiality & $\begin{array}{l}\text { Translation (along } \\
\text { axis) }\end{array}$ & Cylindricity \\
\hline Slider & $\begin{array}{l}\text { Prismatic - } \\
\text { Prismatic }\end{array}$ & $\begin{array}{l}\text { Coaxiality, } \\
\text { Parallelism }\end{array}$ & $\begin{array}{l}\text { Translation (along } \\
\text { axis) }\end{array}$ & $\begin{array}{l}\text { Profile of } \\
\text { Surface }\end{array}$ \\
\hline Hinge $\mathrm{F}$ & $\begin{array}{l}\text { Cylinder - } \\
\text { Cylinder Plane } \\
\text { - Plane }\end{array}$ & $\begin{array}{l}\text { Coaxiality, } \\
\text { Parallelism }\end{array}$ & $\begin{array}{l}\text { Rotation (along } \\
\text { axis) }\end{array}$ & $\begin{array}{l}\text { Circularity, } \\
\text { Flatness }\end{array}$ \\
\hline
\end{tabular}

Each movable element consists of two geometric elements. Each surface of the elements is applied to the appropriate elementary surfaces of TTRS and then a proper form tolerance is defined for each surface based on it. As these contact surfaces also have some geometrical relations between themselves, a geometrical-relation constraint can be also defined between elements using the TTRS method. This geometrical-relation constraint has the role to indicate the required accuracy in that movement. These 
movements occur along the direction in which TTRS has a degree of freedom.

\subsection{Relationship between form tolerance and geometrical-relation constraint}

After predicting the deteriorated status using a geometrical-relation constraint, the result has to be assigned to each geometric element. For this assignment, the relationship between a form tolerance and a geometricalrelation constraint should be defined in a movable element. Figure 5 shows an example of this relationship in the case of a hole and a pin.

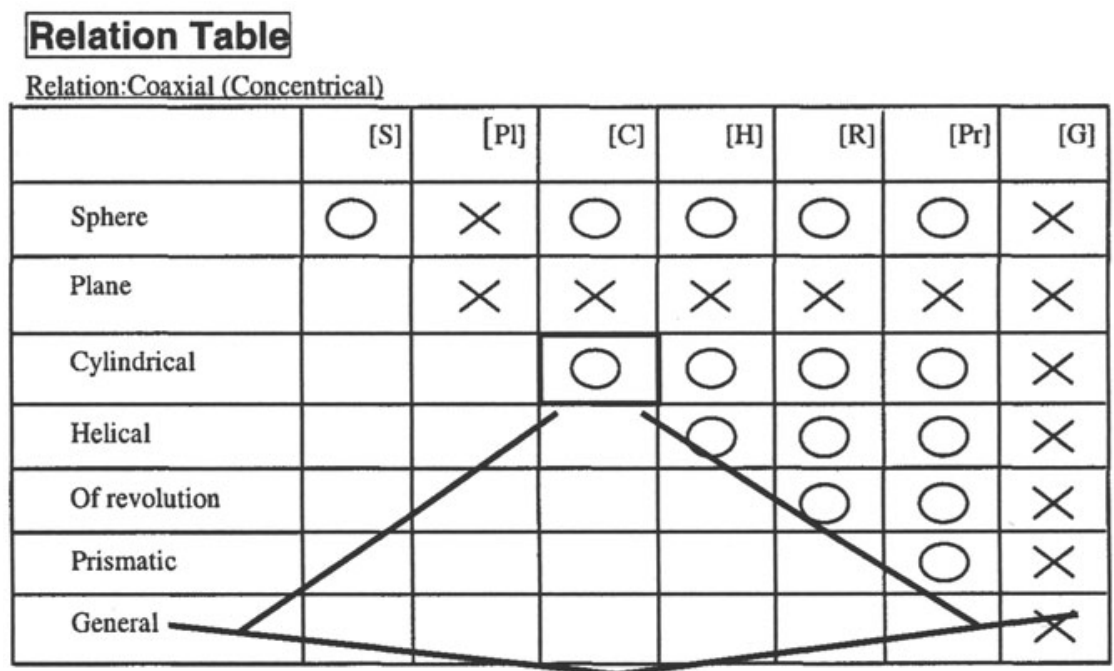

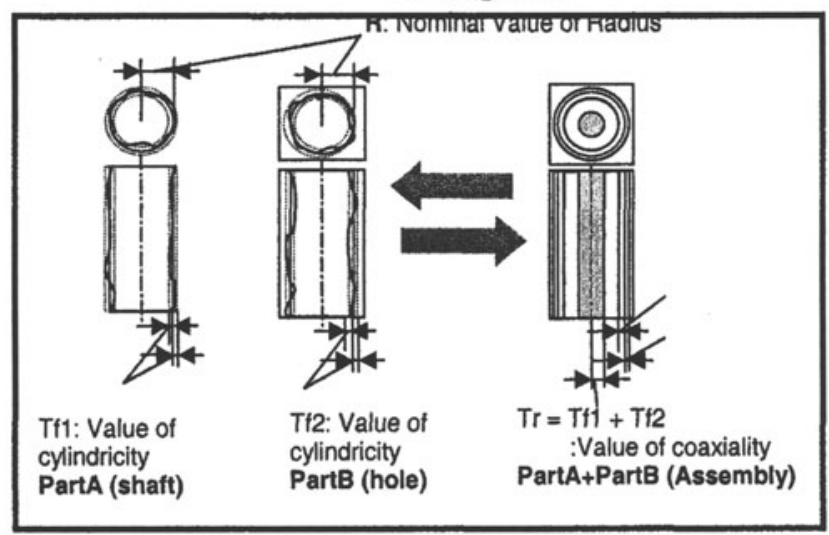

Figure 5. Relationship between form tolerance and geometrical-relation tolerance 
When the geometrical-relation constraint based on the type of form tolerances and its relation is defined, the relational table as shown on the left hand side of Figure 5 is referred to. In this table also describes this relationship between the form tolerances and the geometrical-relation constraint. As both surfaces are cylinders in this example, both part A (shaft) and part $B$ (hole) have the same radius $R$ as a nominal value and these deviations can be restricted by cylindricity. (The values of cylindricities are Tf1 and Tf2 respectively.) Then the shaft and hole are assembled. In the assembly, it is considered that both parts have the shape that provides maximum clearance between them. Under this assumption, the shaft can move freely within the hole due to the clearance. This can be interpreted as the centre of the shaft can move within the cylindrical area whose radius is Tf1+Tf 2 . This means that the value of coaxiality between the shaft and the hole is Tf1+Tf2. This is the relationship between two cylindericities (form tolerance) and a coaxiality (geometrical-relation constraint). Using the above-mentioned relation, the minute deformation of each surface caused by deterioration can be related to the geometrical-relation constraint through the change of form tolerance value.

\subsection{Representation of geometrical quality feature}

Based on the method that was explained in the previous three sections, the geometrical quality feature is implemented in the product model. Figure 6 shows this description and the relation.

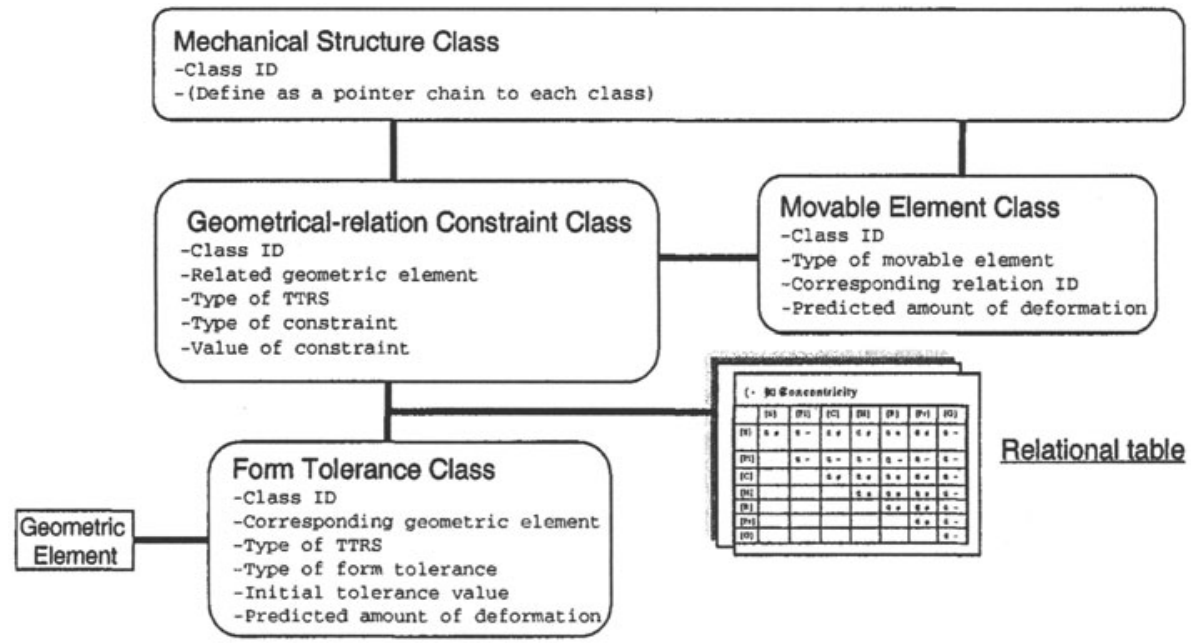

Figure 6. Description of geometrical quality feature 
In the implementation using $\mathrm{C}++$, each element is defined independently as a class object. At first, form tolerances are defined to each geometric element. The type of form tolerance is determined according to the surface type of the geometric element. This form tolerance class has an attribute for adding the amount of deteriorated deformation based on the result of the prediction process. Secondly, a geometrical-relation constraint is defined to geometrical relation between geometric elements. Using the surface type involved the relation and the geometrical relation such as position and orientation, the type of geometrical-relation constraint is specified from the relational table based on the TTRS. If the geometrical-relation constraint is defined between parts and any relative motion occurs in the relation, a movable element has also to be defined to the geometrical-relation constraint. As commonly used movable element classes have already been constructed, the appropriate movable element is applied to the objective movable relation. This class also has an attribute for representing the deteriorated status based on the prediction result. Finally, the whole connection between each class in the product is described as a mechanical structure class. In this class, the connective relation is represented in the form of a pointer chain between each class.

\section{PREDICTION SYSTEM OF DETERIORATION STATUS}

\subsection{Calculation process using cause list}

Using the product model with the geometrical quality feature, the effect of the geometrical accuracy on the functional behaviour of the product is calculated. For this calculation process the cause list is generated based on the mechanical structure class. Figure 7 shows this process and the cause list. 
A mechanical structure class represents the relationship between each element of the geometrical quality feature. In the existing tolerance specification method and tolerance analysis method, a tolerance chain is defined in order to consider the interrelation between each tolerance (Salomons 1995). Therefore this mechanical structure class can be regarded to be the same as a tolerance chain, while, in this paper, functional requirements for satisfying the product function are defined as the geometrical accuracies of assembly relations, behaviour tracks and so on. Corresponding to each functional requirement, a cause list is generated. In this list, the possible sets of the movable elements in which deteriorations occur are given corresponding to a functional requirement to be achieved. In order to decide whether the functional requirement is achieved or not, it is assumed that the threshold value has been given by the designer in advance.

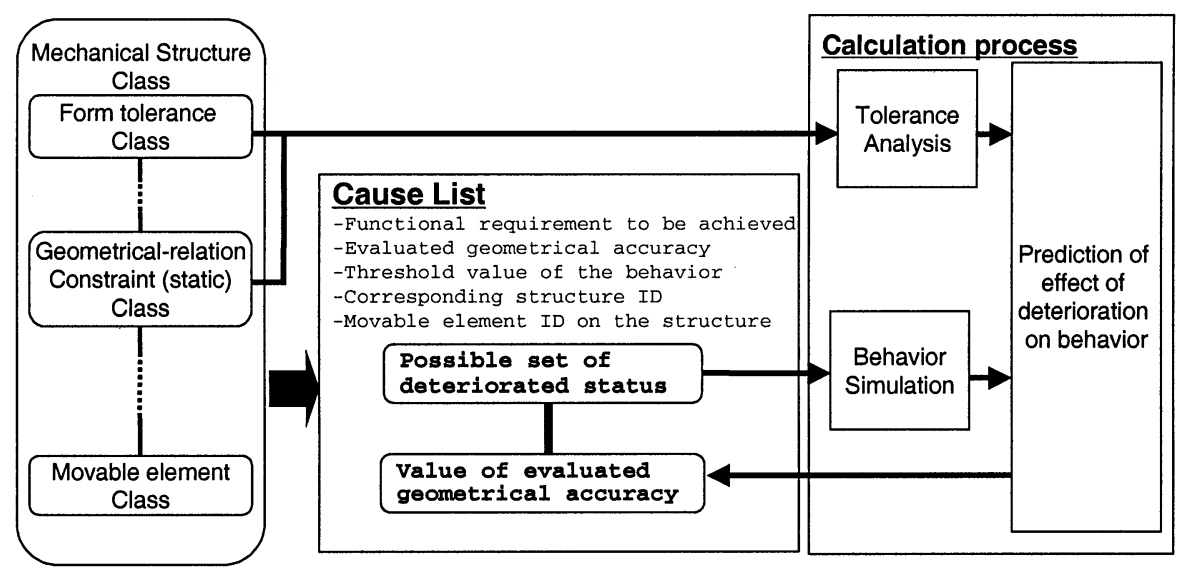

Figure 7. Calculation process using cause list

In the calculation process, each set of deteriorated status given by the cause list is tried with the behaviour simulation. In the behaviour simulation, the value of the corresponding geometrical-relation constraint in the movable element is interpreted as a dimension of the clearance between parts. On the other hand, the other form tolerances and geometrical-relation constraints, which are not related to the deterioration in the use phase, may also have effects on the behaviour due to the initial tolerances. These effects can be calculated using the existing tolerance analysis method in a statistical way. By superimposing the result of behaviour simulation and tolerance analysis, the effect of that set of deteriorated status on the behaviour can be calculated as the value of the corresponding evaluated geometrical accuracy. These results are added to the cause list based on the calculated result. 


\subsection{Prediction process of deteriorated status}

After the calculation process using the cause list mentioned in the previous section, a prediction process for the deteriorated status of parts is proposed as shown in Figure 8.
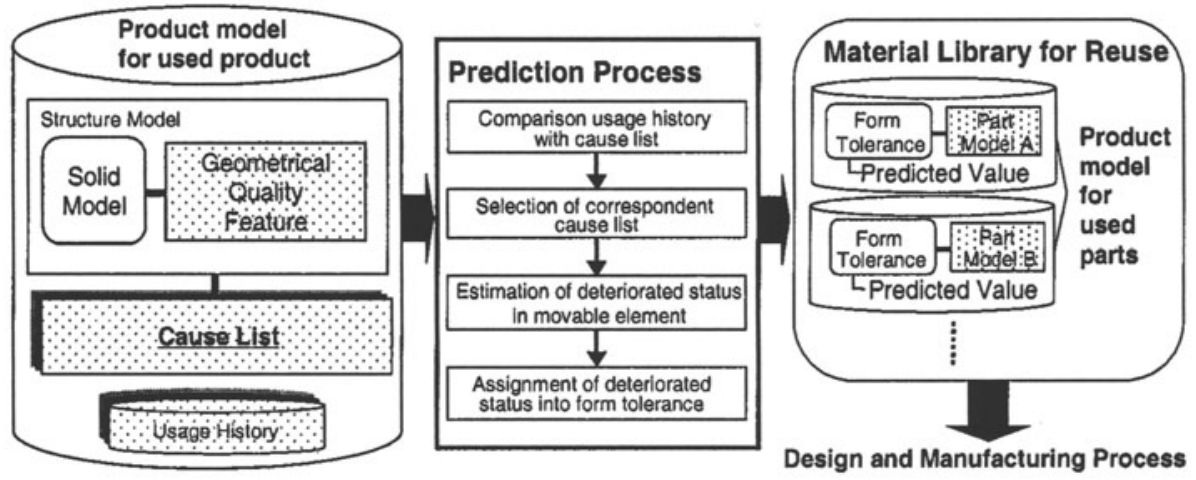

Figure 8. Product Model Based Prediction process for parts deterioration based on the product model

For the prediction process, geometrical quality feature and cause list have been added to the product model for used parts during the product design phase and the use phase. Usage history is also added to the product model during the use phase. This history mainly describes the functional status of the product corresponding to the functional requirement on the cause list.

For the prediction, as the first step the usage history is compared with the threshold value of each functional requirement on the cause list. If a functional requirement to be achieved is found to be satisfied from the usage history, the cause list corresponding to the functional requirement can be selected for predicting the deteriorated status of related movable elements. Based on this, the deteriorated status for each movable element is estimated. Then in each movable element, the deformation is assigned to the form tolerance of each geometric element involved in the movable element. The relationship between geometrical-relation constraint and form tolerance is used for this assignment. Finally, to each used parts model is added the predicted value under the deteriorated status. The used parts model with deteriorated status is added to the material library for the machining phase together with blank material. If it matches the new specification for machining, it can be considered as a candidate for material to be selected. Therefore, each used part can be reused effectively. 


\section{EXAMPLE}

In this chapter, the proposed prediction method is applied to a spindle mechanism. Figure 9 shows this mechanism and its functional requirements and movable elements. In this model, there are two movable elements; left bearing and right bearing. Two functional requirements should be satisfied during usage; the positional error of movement at the edge of the gear both in the axial and radial direction.

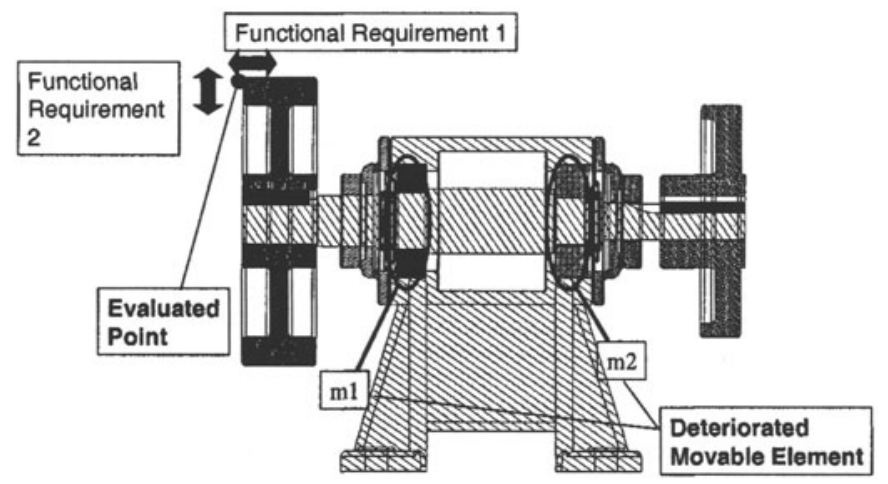

Figure 9. Spindle mechanism

Based on this solid model, both form tolerances and geometrical-relation constraints are added. Figure 10 shows the description of the geometrical quality feature relation of this model. If a movement occurs in the relation, an appropriate movable element is also defined. In this example, a hinge element class is defined between the outer race and inner race of both bearings. By repeatedly generating geometrical quality features for all parts, the mechanical structure class can be defined.

A cause list is then generated based on the mechanical structure and a functional requirement. Then each possible set is checked in the calculation process. In this process, behaviour simulation is executed in order to estimate the evaluated geometrical accuracy for each functional requirement. In the prediction process, if this function requirement is satisfied, the corresponding cause list is selected for predicting the worst deteriorated status. In this case, it is represented as the dimension of clearance in this case, at each bearing element as shown in Figure 11. 


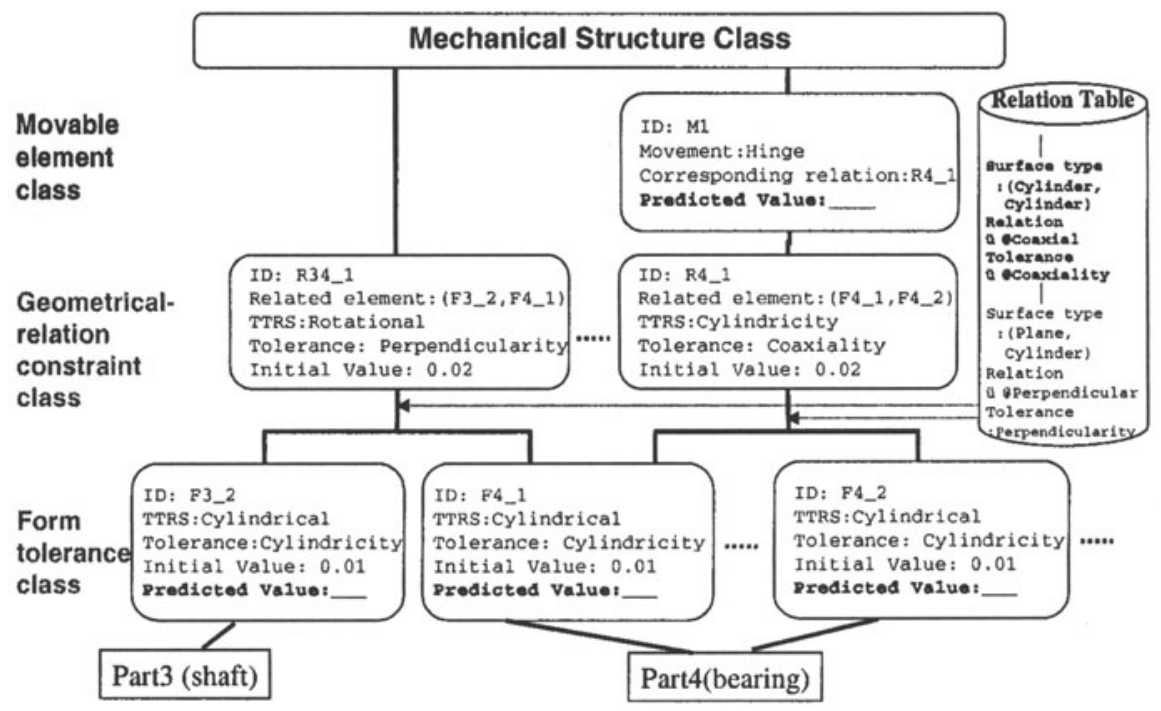

Figure 10. Description of geometrical quality feature of the spindle model

\section{Cause List}

\begin{tabular}{|c|c|c|c|}
\hline \multicolumn{4}{|c|}{ Relation Table } \\
\hline \multicolumn{4}{|c|}{$\begin{array}{l}\text { Mechanism: Spindle } \\
\text { Functional Requirement: fro1, } \\
\text { StartElement: } g 51 \\
\text { Evaluated Element: } g 11 \\
\text { Thereshold Value(fr01): } 0.001 \\
\text { Thereshold Value (fr02): } 0.01\end{array}$} \\
\hline $\mathrm{m}$ & $m e$ & frol & $\mathrm{frO2}$ \\
\hline 0.01 & 0.01 & 0.009 & 0.0084 \\
\hline 0.01 & 0.02 & 0.002927 & 0.00527 \\
\hline 0.01 & 0.03 & 0.009531 & 0.00059 \\
\hline 0.01 & 0.04 & 0.016288 & 0.0001 \\
\hline & & & \\
\hline
\end{tabular}

\begin{tabular}{|c|c|c|c|c|}
\hline \multicolumn{3}{|c|}{ Prediction Result } & \multicolumn{2}{|c|}{$\begin{array}{c}\text { Deteriorated } \\
\text { Value }\end{array}$} \\
\hline $\begin{array}{l}\text { Example } \\
\text { of usage } \\
\text { history }\end{array}$ & \begin{tabular}{|l|} 
Functional \\
Req. 1 \\
Axial Error
\end{tabular} & \begin{tabular}{|l|} 
Functional \\
Req. 2 \\
Radial Error
\end{tabular} & M1 & $\mathrm{m} 2$ \\
\hline Case, P & Satisfied & $\begin{array}{l}\text { Not } \\
\text { Satisfied }\end{array}$ & 0.06 & 0.15 \\
\hline Case2 & $\begin{array}{l}\text { Not } \\
\text { Satisfied }\end{array}$ & Satisfied & 0.12 & 0.13 \\
\hline Case3 & Satisfied & Satisfied & 0.04 & 0.05 \\
\hline
\end{tabular}

Figure 11. Prediction of deteriorated status of movable element 


\section{CONCLUSIONS AND DISCUSSION}

In this paper, a reuse phase for the mechanical parts could be supported successfully by defining a geometrical quality feature in the feature-oriented product model and by predicting the deteriorated status of parts using this feature. The geometrical quality feature was described by semantically extending the concept of the present geometric tolerance. It consisted of form tolerance description, geometrical-relation constraint and the relationship between them. It can be said that this structure has several advantages. The first advantage is that the geometrical quality feature is defined based on the definition of the present geometric tolerance. Therefore, tolerance specification methods, such as the TTRS method, can be applied to that of geometrical quality features. The second advantage is that both of the parts deformation due to deterioration and the change of the assembly relation can be represented using form tolerance descriptions and geometrical-relation constraints respectively. Furthermore by defining the relationship between form tolerance description and geometrical-relation constraint, functional requirements about geometrical accuracy of assembly relations could be interpreted into the form accuracy of physical parts for the refurbishment process.

Using the product model with the geometrical quality feature, a prediction process of deteriorated status could also be proposed. In executing a behaviour simulation for the prediction, geometrical-relation constraints could be treated as geometrical parameters. As a result, the deteriorated status in the movable element be calculated and reflected in terms of the form tolerance. This information is added to the product model and thus helps in the prediction process by comparing the usage history. Finally, the example process for the prediction of deteriorated status could be shown. This prediction process will lead to the creation of an efficient material library including used parts. The material library will make it possible to construct the process planning system including the refurbishment process for used parts. This means that the proposed method permits the construction of feature-oriented product model that could support the realization of a closed product life cycle.

\section{ACKNOWLEDGMENT}

The authors are grateful to Dr. Udo Graefe of the National Research Council of Canada for his helpful assistance with the writing of this paper in English. The authors also thank the Life Cycle Group member in the Kimura Laboratory of the University of Tokyo for a fruitful discussion. 


\section{REFERENCES}

Clement, A., Rivière, A. and Serre, P. (1996). A Declarative Information Model for Functional Requirements, In Kimura, F. (ed.): Computer-aided Tolerancing, Proceedings of the $4^{\text {th }}$ CIRP Design Seminar, pp.3-16.

Krause, F. -L., Kimura, F., Kjellberg, T., and Lu, S. C. -Y. (1993). Product Modelling, In Annals of the CIRP Vol. 42/2/1993, pp. 695-700.

Matsuda, M., and Kimura, F. (1999). A Model Integration Framework for Life Cycle Engineering from the Machining Process Viewpoint, In CIRP ${ }^{\text {th }}$ International Seminar on Life Cycle Engineering, pp. 332-341.

Oyvind Bjorke (1989). Computer-aided Tolerancing, ASME Press.

Salomons, O. (1995). Computer Support In The Design Of Mechanical Products, PhD Thesis of the University of Twente.

Suzuki, H., Matsuda, M. and Kimura, F. (2000). Prediction of Used Parts Deterioration Based on A Tolerance Model, In CIRP $33^{\text {rd }}$ International Seminar on Manufacturing Systems, pp. 149-154. 